\title{
Grading impairment in leprosy
}

\author{
WIM H. VAN BRAKEL*, NAOMI K. REED** \& \\ DARREN S. REED+ \\ *INF Leprosy Project, Pokhara, Nepal \\ **Green Pastures Hospital, Pokhara, Nepal \\ +Western Regional Hospital, Pokhara, Nepal
}

Accepted for publication 28 April 1999

Summary The aim of the paper is to discuss the concept of 'severity grading' in relation to impairment in leprosy, and to describe the use of an impairment sum score, the Eyes, Hands, Feet (EHF) score, as an indicator of the severity and the evolution of impairment over time. The use of an impairment sum score, the EHF score, is illustrated using data on impairment at diagnosis and after a 2-year interval from MB patients released from MDT in the Western Region of Nepal. The WHO 1988 'disability' grading scale ( $0-2$, for both eyes, hands and feet - six sites) was used as a measure of impairment. For the analysis, the WHO grades for the six sites were summed to form an EHF score (minimum 0, maximum 12). The sensitivity to change over time of the EHF score was compared with that of the "method of maximum grades'. Using the 'method of maximum grades', 509/706 patients (72\%) appeared not to have changed in impairment status, compared with only $399(56.5 \%)$ with the EHF score. Improvement or deterioration of impairment status was missed in 113 patients (16\%). In 216/706 patients (30.6\%), the changes detected with the EHF score were bigger than those revealed by the method of maximum grades. The six components of the WHO impairment grading may be added up to form a EHF sum score of impairment. This score can be used to monitor changes in impairment status in individuals or in groups. It should be recorded and reported at least at diagnosis and release from treatment. Reporting could be done as the 'proportion of patients with improved EHF score', 'stable EHF score' and 'EHF score worse', and 'proportion of patients without impairment', 'proportion with WHO grade 1' and 'proportion with WHO grade 2'. It is recommended that the concepts and terminology of the WHO International Classification of Impairments, Activities and Participation (ICIDH-2) be adopted in the field of leprosy, particularly for the areas of prevention of impairment and disability and rehabilitation. The 'WHO disability grade' should be renamed 'WHO impairment grade'.

Correspondence to: Wim H. van Brakel, c/o INF LP, PO Box 5, Pokhara, Nepal. Fax: +977-61-20430, e-mail: wvbrakel@iname.com 


\section{Introduction}

In the rehabilitation of people with impairment, limitations in activities of daily living and/or restrictions in social participation, the question is often not 'how or why did the condition occur', but 'what is the nature and severity of the problem for the person affected?' In other words, a problem-oriented rather than a diagnosis-oriented approach is needed. Rehabilitation needs to be directed at solving the problems experienced by the individual patient. ${ }^{1}$ The same is true in prevention of impairment and disability (POID). To decide what kind of rehabilitation or POID intervention is needed, the health worker needs to 1) assess and classify the problem(s), and 2) grade their severity.

A 'disability classification' for use in leprosy has been advocated by WHO since $1960 .^{2}$ It was developed into its current form in $1988 .{ }^{3}$ The original purpose was to record a baseline 'disability' status to monitor changes during follow-up. ${ }^{2}$ The grading system was therefore quite elaborate. However, by 1988 the main purpose of the grading had changed to being a case finding indicator, to estimate delay in case finding. ${ }^{3}$

Classification helps to decide what kind of problem one is dealing with and what kind of treatment protocol or intervention should be used. It also provides a uniform language for communication between health workers and health centres and for research purposes. ${ }^{4} \mathrm{~A}$ classification is defined as 'a system of concepts (terms) connected by generic relations'. ${ }^{4}$ Each category of a classification is determined by certain predefined characteristics. A very simple example is the multibacillary/paucibacillary (MB/PB) classification in leprosy. ${ }^{5}$

A classification for use in rehabilitation medicine was introduced by WHO in 1980, the International Classification of Impairments, Disabilities and Handicaps (ICIDH). ${ }^{5}$ It defines the concepts of impairment and disability (see below; handicap has been left out because it falls outside the remit of this paper) and then subdivides each into categories and subcategories. Its use in leprosy has been recommended by Brandsma et al., ${ }^{6,7}$ Pönnighaus et $a l^{8}{ }^{8}$ and Smith. ${ }^{9}$ Adoption of the ICIDH framework and terminology in leprosy would greatly help to clarify the current confusion in terms and concepts. The terms 'impairment', 'disability' and 'deformity' are often used interchangeably. Anaesthesia is sometimes referred to as a 'deformity' and a plantar ulcer as a 'disability'. The term 'disability' is used without any reference to rehabilitation.

The ICIDH defines impairment and disability as follows:

Impairment: 'Any loss or abnormality of psychological, physiological, or anatomical structure or function'. 5

Disability: 'Any restriction or lack of ability (resulting from impairment) to perform an activity in the manner of within the range considered normal for a human being'.

Deformity may be defined as 'visible impairment'.

According to these definitions, the well-known 'WHO disability grading scale' does not grade disabilities but impairments. ${ }^{7,9}$ This confusion has also been found with other similar scales. Molenaar et al. noted that the 'neurologic disability score' 'is in fact an impairment measure'. ${ }^{10}$

In 1997, a draft of the revised edition of the ICIDH was published: the ICIDH-2. ${ }^{11}$ In this revised classification, the terms 'disability' and 'handicap' were replaced by the more intuitive and positive terms 'activities' (of daily living) and (social) 'participation'. Problems in these areas are described as 'activity limitation' and 'participation restriction'. We propose that the concepts used in the new ICIDH-2 be used throughout in leprosy work. Besides providing a uniform language for the area of prevention of impairment and disability, it offers 
a client-oriented framework for rehabilitation. In this paper we will use the terms impairment' and 'disability' only according to the above definitions. We will therefore refer to the 'WHO disability grade' as the 'WHO impairment grade'.

Besides classification of the problems a person experiences, it is often important to grade their severity, for example, if progress over time is to be assessed. The purpose of this paper is to discuss the concept of 'severity grading' and to describe the use of an impairment summary score, the Eyes, Hands, Feet (EHF) score, as an indicator of the severity and evolution of impairment over time.

\section{Materials and methods}

To illustrate the use of the EHF score, data are used from a retrospective cohort study on impairment in multibacillary (MB) patients in West Nepal. The methods are described in detail in a separate paper by Reed et al. ${ }^{12}$ In summary, a record review was done of 1082 multibacillary patients (MB) registered between 1980 and 1993 and released from treatment (RFT) between 1983 and 1994 at nine mobile clinic treatment centres in the Western Region. From each patient card, the following information was collected: registration and RFT dates, age, sex, leprosy type, WHO grades at diagnosis and any other recorded at yearly intervals.

The summary score used is the sum of the WHO impairment grades of both eyes, hands and feet (EHF score, minimum '0', maximum '12'). The EHF score was calculated at diagnosis and for annual follow-up examinations for which the patient had attended. To assess whether the patient had improved, stayed the same or deteriorated, the difference in EHF scores between diagnosis and annual follow-ups was calculated. The same was done using the WHO maximum impairment grades.

\section{Results}

Table 1 compares the impairment status as measured with the WHO maximum impairment grade with the result of the $0-12$ grade EHF score. It can be seen that the EHF score gives a much fuller picture of the extent of impairment. Of the 1082 patients, 478 (44.1\%) had no impairment (a score of 0 ) at diagnosis. This left $55.9 \%$ of patients scoring one or more (at least one hand or foot with sensory impairment, or one eye affected by leprosy).

Table 1. Comparison of the maximum WHO impairment grade and the impairment sum score of eyes, hands and feet (EHF score) at diagnosis in $1082 \mathrm{MB}$ patients registered in field clinics in the Western Region, Nepal

\begin{tabular}{|c|c|c|c|c|c|c|c|c|c|c|c|c|c|c|}
\hline \multirow{2}{*}{$\begin{array}{l}\text { Maximum } \\
\text { grade }\end{array}$} & \multicolumn{14}{|c|}{ Summary impairment score (EHF) } \\
\hline & 0 & 1 & 2 & 3 & 4 & 5 & 6 & 7 & 8 & 9 & 10 & 11 & 12 & Total \\
\hline 0 & 478 & & & & & & & & & & & & & 478 \\
\hline 1 & & 87 & 81 & 24 & 85 & & 3 & & & & & & & 280 \\
\hline 2 & & & 100 & 21 & 45 & 44 & 44 & 21 & 32 & 4 & 5 & 2 & 6 & 324 \\
\hline Total & 478 & 87 & 181 & 45 & 130 & 44 & 47 & 21 & 32 & 4 & 5 & 2 & 6 & 1082 \\
\hline
\end{tabular}


Table 2. Changes in maximum WHO impairment grade between time of diagnosis and 2-year follow up in 706 MB patients registered in field clinics in the Western Region, Nepal. The impairment status of the patients who appeared to have remained stable are shown in bold ( 509 or $72 \%)$. Those above the diagonal (top-right segment) deteriorated

\begin{tabular}{|c|c|c|c|c|c|c|c|}
\hline \multirow{2}{*}{$\begin{array}{l}\text { Maximum } \\
\text { grade at } \\
\text { diagnosis }\end{array}$} & \multicolumn{7}{|c|}{ Maximum grade after 2 years } \\
\hline & 0 & $\%$ & 1 & $\%$ & 2 & $\%$ & Total \\
\hline 0 & 279 & $90 \cdot 0$ & 19 & $6 \cdot 1$ & 12 & 3.9 & 310 \\
\hline 1 & 86 & $45 \cdot 7$ & 68 & $36 \cdot 2$ & 34 & $18 \cdot 1$ & 188 \\
\hline 2 & 25 & $12 \cdot 0$ & 21 & $10 \cdot 1$ & 162 & 77.9 & 208 \\
\hline Total & 390 & & 108 & & 208 & & 706 \\
\hline
\end{tabular}

In Table 2, the WHO maximum grades at diagnosis and at 2 years follow-up are crosstabulated. Only 706/1082 patients had a WHO grading recorded at 2 years. 509/706 patients (72\%) appeared not to have changed in impairment status. Table 2 also shows that grade 2 impairment cannot be equated with 'irreversible' impairment: $22 \%$ of patients with a maximum grade of 2 improved during the study period. Table 3 shows a similar comparison for the EHF scores. This time only 399 (56.5\%) appeared unchanged, with only three showing changes in the maximum grade.

Table 4 compares the difference in maximum WHO grade between diagnosis and 2-year follow-up with the difference in EHF sum score during the same period. Of the 509/706 patients who had a stable WHO maximum grade, 113 showed changes in EHF score. Improvement or deterioration of impairment status was thus missed in 113 patients $(16 \%)$

Table 3. Changes in EHF scores between time of diagnosis and 2-year follow up in $706 \mathrm{MB}$ patients registered in field clinics in the Western Region, Nepal. Patients below the diagonal (bottom-left segment) improved; those above deteriorated (top-right segment)

\begin{tabular}{|c|c|c|c|c|c|c|c|c|c|c|c|c|c|c|}
\hline \multirow{2}{*}{$\begin{array}{l}\text { EHF } \\
\text { scores at } \\
\text { diagnosis }\end{array}$} & \multicolumn{13}{|c|}{ EHF scores after 2 years } & \multirow{2}{*}{$\begin{array}{c}\text { Total } \\
\text { no. } \\
\text { patients }\end{array}$} \\
\hline & 0 & 1 & 2 & 3 & 4 & 5 & 6 & 7 & 8 & 9 & 10 & 11 & 12 & \\
\hline 0 & 279 & 7 & 17 & & 6 & & 1 & & & & & & & 310 \\
\hline 1 & 36 & 11 & 11 & & 1 & & & & & & & & & 59 \\
\hline 2 & 41 & 15 & 44 & 7 & 8 & 2 & 3 & 1 & & & & & & 121 \\
\hline 3 & 6 & 5 & 4 & 2 & 4 & 2 & 2 & 1 & & & & & & 26 \\
\hline 4 & 21 & 4 & 12 & 5 & 31 & 7 & 4 & 3 & 2 & & & & & 89 \\
\hline 5 & 2 & 1 & 1 & 3 & 4 & 7 & 6 & 1 & 4 & & & & & 29 \\
\hline 6 & 4 & & 2 & & 5 & 4 & 9 & 3 & 2 & & 1 & & 1 & 31 \\
\hline 7 & & & & & 1 & & 1 & 5 & 2 & 3 & & & & 12 \\
\hline 8 & & & & 2 & & & 2 & 4 & 2 & 8 & & & 1 & 19 \\
\hline 9 & & & & & & & & & & 1 & 1 & & & 2 \\
\hline 10 & & & & & & & & & & & & 1 & & \\
\hline 11 & & & & & & & & & & & 1 & 1 & & \\
\hline 12 & 1 & & & & & & & & & & & & 4 & 5 \\
\hline Total & 390 & 43 & 93 & 18 & 59 & 25 & 34 & 13 & 20 & 1 & 2 & 2 & 6 & 706 \\
\hline
\end{tabular}


Table 4. Comparison of responsiveness to change between the method of the maximum WHO impairment grade and the EHF sum score during 2 years of leprosy treatment in 706 patients in West Nepal. Positive 'difference scores' indicate an improvement; negative scores a worsening. The bold numbers refer to patients in whom the changes detected with the EHF score were bigger than those detected with the maximum WHO impairment score $(216 / 706=30 \cdot 6 \%)$. In $113 / 509$ patients $(22 \%)$ the EHF score detected a change of between 1 and 6 points while the difference between maximum scores was zero

\begin{tabular}{|c|c|c|c|c|c|c|}
\hline \multirow{2}{*}{$\begin{array}{l}\text { Difference } \\
\text { in EHF } \\
\text { score }\end{array}$} & \multicolumn{5}{|c|}{ Difference in maximum WHO impairment grade } & \multirow[b]{2}{*}{ Total } \\
\hline & -2 & -1 & 0 & 1 & 2 & \\
\hline-6 & 1 & & 1 & & & 2 \\
\hline-5 & & 1 & & & & 1 \\
\hline-4 & 1 & 9 & 4 & & & 14 \\
\hline-3 & & 6 & 6 & & & 12 \\
\hline-2 & 10 & 13 & 10 & 1 & 34 & \\
\hline-1 & & 19 & 29 & 1 & & 49 \\
\hline 0 & & 2 & 396 & 1 & & 399 \\
\hline 1 & & 1 & 28 & 48 & & 77 \\
\hline 2 & & 2 & 26 & 31 & 12 & 71 \\
\hline 3 & & & 7 & 2 & 4 & 13 \\
\hline 4 & & & 1 & 22 & 2 & 25 \\
\hline 5 & & & & & 2 & 2 \\
\hline 6 & & & 1 & 1 & 4 & 7 \\
\hline 12 & & & & & 1 & 1 \\
\hline Total & 12 & 53 & 509 & 107 & 25 & 706 \\
\hline
\end{tabular}

with the method of the maximum grade. In $216 / 706$ patients $(30.6 \%)$, the changes detected with the EHF score were bigger than those revealed by the method of maximum grades. One patient recovered completely from having an EHF score of 12 at diagnosis to not having any impairment after 2 years. While there may have been mistakes in his original grading, he was also suffering from ENL reaction at the time. Successful treatment of the reaction may explain this dramatic recovery.

\section{Discussion}

In the current study, the WHO maximum grading system was compared with a simple sum of the WHO impairment grades of both hands, feet and eyes, the EHF score. This idea is not new. De Rijk et al. used a simple sum of the WHO grade of each hand and foot in the AMFES project in Ethiopia. ${ }^{13}$ They omitted the eye grade, because only $5 \%$ of the patients in their sample $(n=286)$ had eye impairment at diagnosis. ${ }^{13}$ In our sample $(n=1082)$, this was $4 \cdot 3 \%$ (95\% CI 3.1-5.6). However, we consider eye impairment very important, particularly in people who may also have hand or foot impairment. Furthermore, the prevalence of eye impairment varies greatly in different patient populations and the score results should be comparable between different projects and centres.

It is clear from the data presented that the EHF score provides a much more detailed picture of the impairment status of individuals than does the maximum WHO grade. Watson remarked that the maximum grade method was not sensitive enough to change. ${ }^{14}$ We have 
shown that the maximum grade method failed to detect changes in impairment status in $16 \%$ of patients compared with when the EHF score was used. In $30.6 \%$, the changes were underestimated when only the maximum grade was used as indicator.

\section{SEVERITY GRADING}

In the management of people with impairment, activity limitations and/or problems with social participation, one often needs to grade the severity of the problem. This will help to assess the urgency of intervention and provide a measure to monitor progress over time. Severity of impairment can be graded by:

1. Assigning different grades for different levels of a given type of impairment.

2. Assigning different weights to different impairments, i.e. a hierarchical system in which certain impairments are more severe than others.

3. Using a summary score of the grades assigned to separate impairments in one individual, i.e. grading the extent of impairment, assuming that more extensive impairment, will be more severe to the patient.

In 1960, WHO adopted a 'classification of disabilities' for use in leprosy, ${ }^{2}$ based on a 6-point scale (0-5), separate for hands, feet and face. The scale graded only impairments according to the ICIDH terminology and was a combination of an impairment severity grading of type 2 above and an impairment classification. Two revisions of this grading system were subsequently published, a 4-point scale in $1970^{15}$ and a 3-point scale in $1988^{3,15}$ These have become widely accepted in leprosy control programmes, although their use is of ten restricted to grading of impairments at diagnosis. Modifications of the WHO grading scale and forms have been proposed by Hasan, ${ }^{16}$ Kulkarni et al., ${ }^{17}$ Watson, ${ }^{14}$ Brandsma et al. ${ }^{18}$ and Thappa. ${ }^{19}$

Two major difficulties faced are:

1. The widely varying needs: a simple system for use in prevention of impairment activities in the field and a detailed system to be used for physical rehabilitation in referral centres.

2. The fact that impairments in leprosy do not always follow set patterns and that the relative severity of different impairments varies among individuals and is related to the resulting activity limitations and/or restriction in social participation.

For example, some people develop sensory impairment before contractures, while others have contractures without sensory impairment. For a person who depends for his daily living on his ability to feel, anaesthesia of his hands may result in a more severe disability than a contracture of one or more fingers. This raises the question whether impairment measures are the most relevant in this situation, or whether activity (disability) measures should be used instead. Discussing outcome measures in peripheral neuropathies, Molenaar et al. stated 'We believe that impairment measures give information on the biological effect of treatment, whereas disability and handicap measures give clinically important and patient relevant information showing whether a treatment improves the patient's functional health'. ${ }^{10}$ In leprosy, physical rehabilitation efforts have mainly centred on prevention and treatment of primary and secondary impairments. ${ }^{20}$ To assess the severity of the impairment in a given individual and to monitor the success of prevention and treatment of impairment, an impairment measure is therefore appropriate. At the same time, measures to assess activities of daily living and social participation relevant for people affected by leprosy should be developed. 
IMPAIRMENT SEVERITY SCORES

It is convenient if the person's condition can be summarized in a single score. Until now, two main approaches have been used: a 'disability' index and the 'maximum WHO disability grade'. The former was first introduced by Bechelli and Martinez Dominguez in 1971. ${ }^{21}$ They proposed three different ways of calculating a disability index (DI) from the grades of the 1970 WHO disability grading. The most commonly used is the DI(2), in which the index is the average of the sums of impairment grades for each hand, foot and eye. ${ }^{22,23}$ The Bechelli DI(2) is a comprehensive summary measure, but, because it is based on the WHO 1960 grading, it requires fairly extensive recording of impairments and is complicated to calculate.

The use of the maximum WHO disability grade to summarize a person's impairment status has been popularized by the inclusion of this indicator in ILEP and WHO statistics. ${ }^{3}$ With this method, the maximum grade of the six individual sites is taken as an overall indicator of the person's impairment status. The validity of the severity weights assigned to different impairments in the WHO grading system, used in both methods, is questionable (Jean Watson, unpublished discussion document on disability grading, 1995). To our knowledge, none of the WHO grading scales has been subjected to reliability testing. This should be urgently done, as an increasing importance is attached to the results of the WHO impairment grading.

\section{SUMMARY SCORES}

The use of sum scores has been criticized by several authors. Van Gijn and Warlow warned strongly against attempting to reconstruct a patient from separate 'functions': 'A patient is more than the sum of his signs' ${ }^{24}$ They argue that numerical changes in (impairment) sum scores of individuals may be meaningless and advocate the use of handicap or disability scales with only few categories, as these are much more meaningful from the patient's point of view. ${ }^{24,25}$ Another criticism of summary scores is that one cannot tell whether a change in score was a major change in one component or only minor changes in several components (Watson, unpublished discussion paper on disability grading, 1995). While this is true, this disadvantage can be reduced by ensuring that only significant changes in components contribute to changes in the sum score.

For example, the EHF score is made up of the components of the WHO impairment grading. A change of one point at any site usually constitutes a major change in impairment status and, therefore, a change of even 1 point in the sum score should be considered clinically significant. In a sum score such as the EHF score, changes may be masked if different components of the score change in opposite directions. For example, if the anaesthetic hand of a patient recovers, but he develops sensory impairment on the sole of one foot, the sum score will show no change. The same occurs when using the maximum grade method.

It should be pointed out that sum scores such as the EHF score are not intended for clinical management of individual patients. They serve as indicators of whether a patient or group of patients is improving, deteriorating or remaining stable. For clinical management, a system such as the Impairment Summary Form, using separate impairment indicators, such as a wound count, a count of sites of bone loss, sites with sensory impairment or changes in voluntary muscle test will be much more useful. ${ }^{26}$

We do not advocate the EHF score as an ideal impairment indicator. It is a simple sum of 
very crude components. Its reliability has to date not been established and its responsiveness to change over time needs further prospective study. However, its main strength lies in the availability of the components. According to a recent survey of the ILEP Medical Commission on prevention of disability activities in ILEP-supported projects, $99 \%$ of the responding projects recorded the WHO impairment grade. ${ }^{27}$ Adding up the six components to form the EHF score makes, in our opinion, much better use of the available data than does the method of maximum grades. Such a score should be calculated at diagnosis, at release from treatment and at other times as needed. Changes should be recorded as 'improved', 'stable' or 'deteriorated' and reported as 'proportion of patients improved', 'proportion of patients remaining stable' and 'proportion of patients deteriorated'. In addition, the 'proportion of patients without impairment', 'proportion with WHO grade 1' and 'proportion with WHO grade 2' should be reported at diagnosis and RFT. Where possible, these data should be based on cohort calculations. Such indicators would provide at least a crude measure of the effectiveness of prevention of impairment activities, until better measures become available.

\section{RECOMMENDATIONS}

1. The concepts and terminology of the ICIDH-2 should be adopted in the field of leprosy, particularly for the areas of prevention of impairment and disability (POID) and rehabilitation.

2. We suggest that the term 'POID' is more appropriate than 'POD' (prevention of disability), given that most activities are aimed at preventing primary or secondary impairments.

3. The 'WHO disability grade' should be renamed 'WHO impairment grade'.

4. Besides the use of the 'proportion of new cases with WHO grade 2' as a case finding indicator, the six components of the WHO impairment grading may be added up to form a $\mathrm{E}(\mathrm{yes}) \mathrm{H}($ ands)F(eet) sum score of impairment. This score can be used to monitor changes in impairment status in individuals or groups of people.

5. The impairment status of patients should be recorded and reported on a cohort basis at least at diagnosis and release from treatment, and more frequently if indicated. This could be done as the 'proportion of patients with improved EHF score', 'stable EHF score' and 'EHF score worse', and 'proportion of patients without impairment', 'proportion with WHO grade 1' and 'proportion with WHO grade 2'.

\section{Acknowledgements}

We are grateful to His Majesty's Government of Nepal for allowing us to work in their country. We would like to thank Dr Alison M. Anderson for helpful comments and suggestions on an earlier version of this manuscript. The work of the International Nepal Fellowship RELEASE Project is dedicated to the glory of God.

\section{References}

1 van Bennekom CAM, Jelles F, Lankhorst GJ. Rehabilitation Activities Profile: the ICIDH as a framework for a problem-oriented assessment method in rehabilitation medicine. Disab. Rehab. 1995; 17: 169-175.

2 WHO Expert Committee on leprosy. Second Report. 189. Technical Report Series, Geneva: WHO, 1960. 
3 WHO Expert Committee on leprosy. Sixth Report. 768. Technical Report Series, Geneva: WHO, 1988.

${ }^{4}$ Brandsma JW, Heerkens YF, van Ravensberg CD. Impairment and disabilities in hand therapy: the necessity of a uniform terminology for communication and research purposes. J. Hand Ther. 1993; Oct-Dec: 252-258.

5 World Health Organisation. International classification of impairments, disabilities and handicaps. Geneva: World Health Organisation, 1980.

6 Brandsma JW. Terminology in leprosy rehabilitation and guidelines for nerve function assessment. Trop. Geograph. Med. 1994; 46: 88-92.

7 Brandsma JW, Heerkens YF, Lakerveld-Heyl K, van Ravensberg CD. The international classification of impairments, disabilities and handicaps in leprosy-control projects. Lepr. Rev. 1992; 63: 337-344.

${ }^{8}$ Pönnighaus IM, Boerrigter G, Fine PE, Pönnighaus JM, Russell J. Disabilities in leprosy patients ascertained in a total population survey in Karonga District, northern Malawi. Lepr. Rev. 1990; 61: 366-374.

9 Smith WCS. The epidemiology of disability in leprosy including risk factors. Lepr. Rev. 1992; 63: 23s-30s.

10 Molenaar DSM, de Haan R, Vermeulen M. Impairment, disability, or handicap in peripheral neuropathy: analysis in the use of outcome measures in clinical trials in patients with peripheral neuropathies. J. Neurol. Neurosurg. Psychiatry 1995; 59: 165-169.

11 World Health Organisation. ICIDH-2: International Classification of Impairments, Activities and Participation. A manual of disablement and functioning. Beta-1 draft for field trials. Geneva: World Health Organisation, 1997.

12 Reed NK, van Brakel WH, Reed DS. Progress of impairment scores following commencement of chemotherapy in multibacillary leprosy patients. Int. J. Lepr. Other Mycobact. Dis. 1997; 65: 328-336.

13 de Rijk AJ, Gabre S, Byass P, Berhanu T. Field evaluation of WHO-MDT of fixed duration, at ALERT, Ethiopia: the AMFES project - I MDT course completion, case holding and another score for disability grading. Lepr. Rev. 1994; 65: 305-319.

14 Watson JM. WHO disability grading (letter). Lepr. Rev. 1985; 56: 172-175.

15 WHO Expert Committee on leprosy. Fourth Report. 459. 1970. Technical Report Series, Geneva: WHO.

16 Hasan S. An appraisal of use of the classification of disabilities resulting from leprosy in field work and suggestions for improvement. Lepr. Ind. 1982; 54: 135-142.

17 Kulkarni UP, Kulkarni VN, Jogaikar DG. Classification on disabilities as followed by Poona District Leprosy Committee. Ind. J. Lepr. 1984; 56: 269-279.

18 Brandsma JW, Tjepkema T, de Jong N, Tjepkema A. Disability grading in leprosy, suggested modifications to the WHO disability grading form. Lepr. Rev. 1986; 57: 361-369.

19 Thappa DM. Disability grading in leprosy: current status. Ind. J. Lepr. 1994; 66: 299-306.

20 Srinivasan H. Prevention of disabilities in patients with leprosy. A practical guide. Geneva: World Health Organisation, 1993.

21 Bechelli LM, Martinez Dominguez V. Disability index for leprosy patients. Bull. World Hlth Org. 1971; 44: 709713

22 Smith WCS, Parkhe SM. Disability assessment as a measure of progress in leprosy control. Lepr. Rev. 1986; 57: 251-259.

23 Thappa DM, Kaur S, Sharma VK. Disability index of hands and feet in patients attending an urban leprosy clinic. Ind. J. Lepr. 1990; 62: 328-337.

24 van Gijn J, Warlow CP. Down with stroke scales! Cerebrovasc. Dis. 1992; 2: 244-246.

25 van Gijn J. Measurement of outcome in stroke prevention trials. Cerebrovasc. Dis. 1992; 2: 23-34.

26 ILEP. Prevention of disability. Guidelines for leprosy control programmes. London: ILEP, 1993.

27 ILEP. Prevention of disability in leprosy. Issue no. 8. 12-95. Medical Bulletin. ILEP Medical Commission. 